\title{
An Implicit Lagrangian Code for Spherically Symmetric General Relativistic Hydrodynamics with an Approximate Riemann Solver
}

\author{
Shoichi Yamada ${ }^{1}$ \\ Max-Planck-Institut für Astrophysik
}

\begin{abstract}
An implicit Lagrangian hydrodynamics code for general relativistic spherical collapse is presented. This scheme is based on an approximate linearized Riemann solver (Roe type scheme) and needs no artificial viscosity. This code is aimed especially at the calculation of the late phase of collapse-driven supernovae and the nascent neutron star, where there is a remarkable contrast between the dynamical time scale of the proto-neutron star and the diffusion time scale of neutrinos, without such severe limitation of the Courant condition at the center of the neutron star. Several standard test calculations have been done and their results show (1) this code captures the shock wave accurately, though some erroneous jumps of specific internal energy are found at the contact discontinuity in the shock tube problems. (2) The scheme shows no instability even if we choose the Courant number larger than 1. (3) However, the Courant number should be kept below $\sim 0.2$ at the shock position so that the shock can be resolved with a few meshes. (4) The scheme reproduces the well known analytic solutions to the point blast explosion, the gravitational collapse of the uniform gas with $\gamma=4 / 3$ and the general relativistic collapse of uniform dust. Two other adiabatic simulations have also been done in order to test the performance of the code in the context of the collapse-driven supernovae. It is found that the time step can be extended far beyond the Courant limitation at the center of the neutron star. The details of the scheme and the results of these test calculations are discussed.
\end{abstract}

Subject headings: Supernovae - general relativity — hydrodynamics numerical simulation

\footnotetext{
${ }^{1}$ Department of Physics, Graduate School of Science, The University of Tokyo
} 


\section{INTRODUCTION}

Since the pioneering work by Colgate and White (1966), detailed studies of the dynamics of collapse-driven supernovae have been done mainly with numerical simulations (Bethe 1990, Müller 1991, and references therein). One of the difficulties in so doing is the remarkable contrast between the dynamical time scale of the nascent neutron star ( $\lesssim 1 \mathrm{msec}$ ) and the diffusion time scale of neutrinos $(\sim 1 \mathrm{sec})$. If we want to simulate the whole scenario of the collapse-driven supernovae, these time scales should be treated simultaneously. Since the typical time scale of weak interactions $\left(\sim 10^{-7} \mathrm{sec}\right)$ is much smaller than the dynamical time scale, the neutrino transfer has been treated in implicit ways in general. The hydrodynamics, however, has been calculated chiefly by explicit schemes, so that the time steps are restricted by the Courant condition and a large number of integration steps are required to simulate the late stage of collapse-driven supernovae. Two approaches have been conceived to overcome such severely limited time steps. The first one is to implement an algorithm of individual time steps in the explicit difference scheme (Bowers \& Wilson 1991), in which the time steps at different positions are allowed to be different from one another and are determined from the local Courant condition. The advantage of this approach is that it is easily applied to multi-dimensional simulations. On the other hand, the implicit difference scheme is another possibility, since the time step is not restricted by the Courant condition, though the required accuracy of calculation will limit the time step. The disadvantage of this approach is that the number of operations per

each time step becomes much larger than in the corresponding explicit scheme, particularly in multi-dimensional simulations. Hence, it is of no use unless we can take time steps large enough to make up for its larger operation number. However, note that the nascent neutron star, whose dynamical time scale limits the time step in the explicit schemes, evolves in a nearly hydrostatic manner so that it might be possible to take large enough time steps in this case. Note also that an advantage of implicit hydrodynamic schemes in the simulation of collapse-drive supernovae is that the neutrino transfer schemes are generally coded in implicit fashions so that the hydrodynamic parts are easily incorporated into the neutrino parts.

The purpose of this paper is to provide such an implicit scheme from the latter stand point. Such attempts have already been made by Schinder et al. (1988) and Swesty (1995). Their schemes use almost the same equation set as that of May \& White (1967), which has been frequently used in explicit general relativistic calculations (note, however, that Schinder et al. extended it to polar-slicing coordinates). Their codes also need artificial viscosities. On the other hand, for the present scheme we tried to apply a so-called Godunov-type differencing method (Hirsch 1990 and references therein), which has been extensively utilized for the multi-dimensional hydrodynamic simulations these days. Since 
we have evaluated the pressure and velocity at a cell interface using the solution of the linearized advection equations, this code is an extension of Roe's scheme. Thanks to the numerical diffusion induced implicitly by finite differencing, this code needs no artificial viscosity like most of the recent elaborate Euler schemes. In addition, it is quite robust. In fact, as shown later, the calculation does not collapse even if we take time steps much larger than the Courant limit, (though the results become less accurate).

This paper is not aiming at the general application of Roe's scheme to general relativistic hydrodynamics (Eulderink \& Mellema 1995, Romero et al. 1995). The coordinates and the metric we use here are fully reduced ones under the assumption of spherical symmetry so that the degree of time slicing does not remain at all. The equation set is also so chosen that they look similar to the nonrelativistic counter parts in these coordinates. Instead, the final goal of this project is to study the neutrino physics in collapse-driven supernovae by combining this code with sophisticated neutrino transfer codes. Recently, many researchers in this field have considered that the key factors of successful explosions are neutrino and multi-dimensional hydrodynamical effects such as convection (Herant et al. 1994, Burrows et al. 1995, Janka \& Müller 1995, Shimizu et al. 1994). It is true that the spherical simulations cannot treat such multi-dimensional effects properly, but we think that they can play a complementary role to multi-dimensional simulations since multi-dimensional simulations use a more approximate treatment of neutrino transport than in spherical calculations. At present we are trying to incorporate this hydrodynamical code into the multi-group flux-limited diffusion code (Suzuki 1990). The coding of the Boltzmann solver has also been undertaken. This paper is the first step of the project.

The organization of this paper is as follows. The details of the formulations and the schemes are described in the next section. The results of the test calculations are shown in the section 3. Concluding remarks are provided in the final section.

\subsection{Basic Equations}

Spherical symmetry of the system is assumed and the following form of the metric is used:

$$
d s^{2}=e^{2 \phi(t, m)} c^{2} d t^{2}-e^{2 \lambda(t, m)}\left(\frac{G}{c^{2}}\right)^{2} d m^{2}-r^{2}(t, m)\left(d \theta^{2}+\sin ^{2} \theta d \phi^{2}\right)
$$

In the above formula $c$ and $G$ are the velocity of light and the gravitational constant, respectively, which are taken to be unity in the following equations. $t$ is the coordinate time and $m$ is the baryon mass coordinate which is related to the circumference radius $r$ 
through the conservation law of baryon mass as described below. This form of the metric is so simple that it has been frequently adopted in the spherically symmetric simulations thus far. However, this way of time slicing is not suitable for the study of black hole formation (see Schinder et al. 1988). Since our purpose is to investigate the supernova dynamics leading to proto-neutron star formation, that is not a serious problem. The basic equations consist of the Einstein equations obtained from the above metric:

$$
G^{\mu \nu}=8 \pi T^{\mu \nu}
$$

and the Euler equations:

$$
\begin{aligned}
\nabla_{\nu} T^{\mu \nu} & =0 \\
T^{\mu \nu} & \equiv\left\{\rho_{b}(1+\varepsilon)+p\right\} u^{\mu} u^{\nu}-p g^{\mu \nu}
\end{aligned}
$$

with the baryon number conservation equation:

$$
\nabla_{\mu}\left(\rho_{b} u^{\mu}\right)=0
$$

and the evolution equation of electron fraction (see below). Here $G^{\mu \nu}$ and $T^{\mu \nu}$ are the Einstein tensor and the energy-momentum tensor, respectively. $\rho_{b}$ is the baryon mass density and $u^{\mu}$ is the four velocity of the matter. $\varepsilon$ and $p$ are the specific internal energy density and the matter pressure, respectively. $g^{\mu \nu}$ is the inverse of the metric tensor $g_{\mu \nu}$. In addition to these equations, the neutrino transfer equations should be implemented finally, but they are dropped in this paper. For this reason, the source term which evolves the electron fraction is not included in the present calculations. First I will write down the hydrodynamic equations with those representing the baryon number conservation and the evolution of electron fraction. They are formulated as follows:

$$
\begin{aligned}
e^{-\phi} \frac{\partial \tau}{\partial t} & =\frac{1}{\Gamma} \frac{\partial}{\partial m}\left(4 \pi r^{2} U\right)-\frac{4 \pi r^{2} \tau F_{\nu}}{\Gamma r} \\
e^{-\phi} \frac{\partial U}{\partial t} & =-\frac{\Gamma}{h} 4 \pi r^{2}\left(\frac{\partial p}{\partial m}+\frac{\tau q}{4 \pi r^{2}}\right)-\frac{\tilde{m}}{r^{2}}-4 \pi r\left(p+p_{\nu}\right) \\
e^{-\phi} \frac{\partial \varepsilon}{\partial t} & =-\frac{1}{\Gamma}\left\{p \frac{\partial}{\partial m}\left(4 \pi r^{2} U\right)-p \frac{4 \pi r^{2} \tau F_{\nu}}{r}\right\}-\tau Q \\
& =-\frac{1}{\Gamma} \frac{\partial}{\partial m}\left(4 \pi r^{2} p U\right)-\frac{h}{\Gamma^{2}} e^{-\phi} \frac{\partial}{\partial t}\left(\frac{1}{2} U^{2}\right)+\frac{h}{\Gamma^{2}} \tilde{m} e^{-\phi} \frac{\partial}{\partial t}\left(\frac{1}{r}\right) \\
e^{-\phi} \frac{\partial Y_{e}}{\partial t} & =-\frac{h U}{\Gamma^{2}} 2 \pi e^{-\phi} \frac{\partial r^{2}}{\partial t}\left(p+p_{\nu}\right)-\frac{1}{\Gamma} \tau U q+\frac{p}{\Gamma} 4 \pi r \tau F_{\nu}-\tau Q \\
\frac{\partial\left(\frac{4}{3} \pi r^{3}\right)}{\partial m} & =\Gamma\left(\frac{\delta f_{\nu}}{\delta \lambda}\right)_{\text {coll. }} \frac{d^{3} p}{p^{0}}
\end{aligned}
$$


In the above equations, $\tau \equiv \frac{1}{\rho_{b}}$ is the inverse of the baryon mass density, and $U \equiv e^{-\phi} \frac{\partial r}{\partial t} \equiv D_{t} r$ is the radial fluid velocity. $\Gamma$ is the general relativistic gamma factor which is defined below (equation (16)) and is solved simultaneously. $h$ is the specific enthalpy which is also defined below (equation (19)) and is treated as the dependent variable to be solved. $\tilde{m}(r)$ is the gravitational mass inside the radius of $r$ and should be distinguished from the baryon mass coordinate $m$. The second representation of the energy conservation (9) looks peculiar. This form of the equation declares explicitly that we get the internal energy density by solving the equation of the total energy conservation of the fluid (in the Newtonian limit) and then extracting the kinetic energy and the gravitational energy from it rather than using the simpler form (8) which is just the first law of thermodynamics. Both forms of the energy equation are tried in the shock tube calculations and it is found that the latter form (9) better reproduces the Rankine-Hugoniot relation. This is a well known fact in the Eulerian numerical simulations. Note that the artificial viscosity is not necessary in both cases for the finite difference scheme described below. $F_{\nu}, p_{\nu}, q, Q$ and $f_{\nu}$ are quantities related with neutrinos. They are defined as follows:

$$
\begin{aligned}
T_{\nu}^{\alpha \beta} & \equiv \rho_{b} E_{\nu} u^{\alpha} u^{\beta}+F_{\nu}^{\alpha} u^{\beta}+u^{\alpha} F_{\nu}^{\beta}+p_{\nu}^{\alpha \beta} \\
Q^{\alpha} & \equiv T_{\nu}^{\alpha \beta} ; \equiv\left(e^{-\phi} Q, e^{-\lambda} q, 0,0\right) \\
& =\int\left(\frac{\delta f_{\nu}}{\delta \lambda}\right)_{\text {coll. }} p^{\alpha} \frac{d^{3} p}{p^{0}} .
\end{aligned}
$$

$T_{\nu}^{\alpha \beta}$ is the energy-momentum tensor of neutrinos and $\rho_{b}$ is the baryon mass density. $E_{\nu}$, $F_{\nu}^{\alpha}$ and $p_{\nu}^{\alpha \beta}$ are, respectively, the energy density, the flux vector and the stress tensor of neutrinos. The right hand sides of equations (10) and (14) are the phase space integrals of the collision term in the neutrino Boltzmann equations. Although these terms are included in the above equations for completeness, they are omitted in the present calculations. In particular, the right hand side of equation $(10)$ is set to be zero, so that $Y_{e}=$ const. in all of the computations below. Equation (11) relates the radius and the baryon mass inside it and is obtained from the combination of the baryon number conservation equation (5) and the definition of $\Gamma$ below (equation (16)). This equation is used to determine $r(m)$ of the next step instead of $D_{t} r=U$, which has been often used so far. It should be noted that the above equations are very similar to the Newtonian counterparts. As a result, the difference scheme developed for the Newtonian system can be applied to the above general relativistic system. The rest of the basic equations specify the metric components $\lambda(t, m)$ and $\phi(t, m)$, the gamma factor $\Gamma(t, m)$, the gravitational mass $\tilde{m}(t, m)$ and the specific enthalpy $h(t, m)$. These equations are:

$$
e^{\lambda}=\frac{1}{\Gamma} \frac{\partial r}{\partial m}
$$




$$
\begin{aligned}
\Gamma^{2} & =1+u^{2}-\frac{2 \tilde{m}}{r} \\
h \frac{\partial \phi}{\partial m} & =-\tau \frac{\partial p}{\partial m}-\frac{\tau^{2} q}{4 \pi r^{2}} \\
\frac{\partial \tilde{m}}{\partial t} & =-\left(p+p_{\nu}\right) \frac{\partial}{\partial t}\left(\frac{4}{3} \pi r^{3}\right)-4 \pi r^{2} e^{\phi} \Gamma F_{\nu} \\
h & =1+\varepsilon+p \tau .
\end{aligned}
$$

As is clear, these are not evolutionary equations except for equation (18), but are constraint equations which must be satisfied at each time step. Since the present scheme is written in the implicit form, these constraints are always satisfied within the error for convergence of iteration. The equation for the gravitational mass $\tilde{m}$ can be written in the constraint form as well:

$$
\frac{\partial \tilde{m}}{\partial m}=4 \pi r^{2}\left[\rho_{b}\left(1+\varepsilon+E_{\nu}\right)+\frac{U F_{\nu}}{\Gamma}\right] \frac{\partial r}{\partial m} .
$$

However, equation (18) is adopted here rather than (20), since it explicitly guarantees the total energy conservation $\tilde{m}(t, R)=$ const., where $R$ is the stellar core radius, as long as the energy loss due to the emission of neutrinos is disregarded. The constraint equation (20) is used for the check of the accuracy of calculations. The terms concerning the interactions of neutrinos are included in the above equations also, but are dropped in the present simulations again. Equation (19) is nothing but the definition of specific enthalpy. Usually, this expression is expanded in the preceding equations. However, it is treated as one of the dependent variables to be solved simultaneously in this code, since it avoids the repeated appearance of the derivatives of $h$ in the matrix elements. This treatment means that the specific enthalpy does not satisfy the above definition strictly during the iteration process. This did not cause any serious problem in the test calculations shown below. Moreover it increases CPU time very little. It should be noticed that the most time consuming part in the radiative hydrodynamics code is the neutrino transfer subroutine. In order to integrate equation (17) we must specify the boundary condition for $\phi$. It is done so that the metric in the stellar core coincides at the surface of the stellar core with the Schwarzschild metric for a spherically symmetric vacuum:

$$
e^{\phi_{s}}=\left(1-\frac{2 \tilde{m}_{s}}{r_{s}}\right)\left(1+U_{s}^{2}-\frac{2 \tilde{m}_{s}}{r_{s}}\right)^{-\frac{1}{2}}
$$

where the subscript " $s$ " means the variables are evaluated at the surface of the stellar core. Strictly speaking, the metric outside the stellar core is not the Schwarzschild metric because neutrinos exist there. For simplicity, however, the above approximation is adopted in the following calculations. To summarize, the basic equations to be solved are equations (6) 
through (11) and (15) through (19). The latter form (9) is used for the energy conservation equation as stated above. The boundary condition is given as equation (21). These equations are differenced in the implicit method described in the next section. The resulting equations are the simultaneous equations consisting of $10 \times N$ (mesh number) equations. The matrix to be inversed has a block-tridiagonal form.

\subsection{The Implicit Differencing Scheme}

All the dependent variables except $r, \phi$ and $\tilde{m}$ are defined at the mesh centers as in most of the recent Eulerian schemes. The values at the cell interfaces for pressure and velocity are calculated by using the solutions of the linearized Riemann problems as described below. $r, \phi$ and $\tilde{m}$ are placed at the edges of meshes in order to simplify the integrals (equation (17)) and the implementation of the boundary condition (equation (21)). The superscripts and the subscripts attached to the variables in the following difference equations are representing the time step and the mesh number, respectively. It is assumed that the mesh centers are specified by the lower case subscript like " $i$ ", on the other hand to the interfaces attached are the uppercase subscript like " $I$ ", and that all these subscripts are integers. It follows that $i$-th mesh center $(i=1 \sim N)$ is located between the $(I-1)$-th and $I$-th interfaces $(I=1 \sim N)$. However, there is one exception. $\phi_{I}$ 's are defined on the $(I-1)$-th interfaces. This is because the boundary condition of $\phi$ (equation (21)) is specified on the surface of the iron core ( $N$-th interface). This is not the case for $r$ and $\tilde{m}$, for which the inner boundary conditions: $r_{c}=0$ and $\tilde{m}_{c}=0$ are assumed implicitly and we don't have to solve them.

Paying attention to these assumptions, we will first see the finite differenced equations regarding the $i$-th mesh and $I$-th interface (but $(I-1)$-th interface only for $\phi$ ):

$$
\begin{aligned}
0 & =\frac{\Delta m_{i}}{4 \pi \Delta t} e^{-\ll \phi \gg_{i}^{n+\frac{1}{2}}}\left(\tau_{i}^{n+1}-\tau_{i}^{n}\right)-\frac{1}{\left\langle\Gamma>_{i}^{n+\frac{1}{2}}\right.}\left\{\left(\left\langle r>_{I}^{n+\frac{1}{2}}\right)^{2} u_{I}^{n+1}-\left(\left\langle r>_{I-1}^{n+\frac{1}{2}}\right)^{2} u_{I-1}^{n+1}\right\}\right.\right. \\
0 & =\frac{\Delta m_{i}}{4 \pi \Delta t} e^{-\ll \phi \gg_{i}^{n+\frac{1}{2}}}\left(U_{i}^{n+1}-U_{i}^{n}\right)+\frac{\left\langle\Gamma>_{i}^{n+\frac{1}{2}}\right.}{\left\langle h>_{i}^{n+\frac{1}{2}}\right.}\left\{\left\langle r>_{i}^{n+\frac{1}{2}}\right\}^{2}\left(p_{I}^{n+1}-p_{I-1}^{n+1}\right)\right. \\
& +\frac{\Delta m_{i}}{4 \pi} \frac{\ll \tilde{m} \gg_{i}^{n+\frac{1}{2}}}{\bar{r}_{i}^{n} \bar{r}_{i}^{n+1}}+\Delta m_{i} \overline{\left\langle r>_{i}^{n+\frac{1}{2}}\right.}\left\langle p>_{i}^{n+\frac{1}{2}}\right.
\end{aligned}
$$




$$
\begin{aligned}
& 0=\frac{\Delta m_{i}}{4 \pi \Delta t} e^{-\ll \phi \gg_{i}^{n+\frac{1}{2}}}\left(\varepsilon_{i}^{n+1}-\varepsilon_{i}^{n}\right) \\
& +\frac{1}{<\Gamma>_{i}^{n+\frac{1}{2}}}\left\{\left(<r>_{I}^{n+\frac{1}{2}}\right)^{2} u_{I}^{n+1} p_{I}^{n+1}-\left(<r>_{I-1}^{n+\frac{1}{2}}\right)^{2} u_{I-1}^{n+1} p_{I-1}^{n+1}\right\} \\
& +\frac{\left\langle h>_{i}^{n+\frac{1}{2}}\right.}{\Gamma_{i}^{n} \Gamma_{i}^{n+1}} e^{-\ll \phi \gg_{i}^{n+\frac{1}{2}}} \frac{\Delta m_{i}}{4 \pi \Delta t} \frac{1}{2}\left\{\left(U_{i}^{n+1}\right)^{2}-\left(U_{i}^{n}\right)^{2}\right\} \\
& -\frac{\left\langle h>_{i}^{n+\frac{1}{2}}\right.}{\Gamma_{i}^{n} \Gamma_{i}^{n+1}} e^{-\ll \phi \gg_{i}^{n+\frac{1}{2}}} \frac{\Delta m_{i}}{4 \pi \Delta t} \ll \tilde{m} \gg_{i}^{n+\frac{1}{2}}\left(\frac{1}{\bar{r}_{i}^{n+1}}-\frac{1}{\bar{r}_{i}^{n+1}}\right) \\
& +\frac{<h>_{i}^{n+\frac{1}{2}}}{\Gamma_{i}^{n} \Gamma_{i}^{n+1}} \frac{\Delta m_{i}}{\Delta t} e^{-\ll \phi \gg_{i}^{n+\frac{1}{2}}} \frac{1}{2}\left\{\left(\bar{r}_{i}^{n+1}\right)^{2}-\left(\bar{r}_{i}^{n}\right)^{2}\right\}<p>_{i}^{n+\frac{1}{2}} \\
& 0=\frac{\Delta m_{i}}{4 \pi \Delta t} e^{-\ll \phi \gg_{i}^{n+\frac{1}{2}}}\left(Y_{e}^{n+1}{ }_{i}-Y_{e i}^{n}\right) \\
& 0=\frac{4 \pi}{3}\left\{\left(r_{I}^{n+1}\right)^{3}-\left(r_{I-1}^{n+1}\right)^{3}\right\}-\Gamma_{i}^{n+1} \tau_{i}^{n+1} \Delta m_{i} \\
& 0=e^{\lambda_{i}^{n+1}}-\frac{1}{\Delta m_{i} \Gamma_{i}^{n+1}}\left(r_{I}^{n+1}-r_{I-1}^{n+1}\right) \\
& 0=\left(\Gamma_{i}^{n+1}\right)^{2}-\left\{1+\left(U_{i}^{n+1}\right)^{2}-\frac{\tilde{m}_{I}^{n+1}+\tilde{m}_{I-1}^{n+1}}{\bar{r}_{i}^{n+1}}\right\} \\
& 0=h_{i}^{n+1}\left(\phi_{I+1}^{n+1}-\phi_{I}^{n+1}\right)+\tau_{i}^{n+1}\left(p_{I}^{n+1}-p_{I-1}^{n+1}\right) \\
& 0=\tilde{m}_{I}^{n+1}-\tilde{m}_{I}^{n}+p_{I}^{n+1} \frac{4 \pi}{3}\left\{\left(r_{I}^{n+1}\right)^{3}-\left(r_{I}^{n}\right)^{3}\right\} \\
& 0=h_{i}^{n+1}-\left(1+\varepsilon_{i}^{n+1}+\tau_{i}^{n+1} p_{i}^{n+1}\right) .
\end{aligned}
$$

In the above equations it is supposed that the time step " $n$ " corresponds to the present time, where all the values of the dependent variables are known. The above finite differenced equations form nonlinearly coupled simultaneous equations. The Newton-Raphson scheme is used to solve these equations. Some averaging procedures are abbreviated like $\langle\cdots\rangle$, $\ll \cdots \gg$ and $\cdots$. Their definitions are as follows:

$$
\begin{aligned}
<X>_{i}^{n+\frac{1}{2}} & \equiv \frac{X_{i}^{n+1}+X_{i}^{n}}{2} \\
\ll Y \gg_{i}^{n+\frac{1}{2}} & \equiv \frac{Y_{I}^{n+1}+Y_{I+1}^{n+1}+Y_{I}^{n}+Y_{I+1}^{n}}{4} \\
\bar{Z}_{i+\frac{1}{2}}^{n} & \equiv\left\{\frac{\left(Z_{I}^{n}\right)^{3}+\left(Z_{I-1}^{n}\right)^{3}}{2}\right\}^{\frac{1}{3}},
\end{aligned}
$$


where $X$ is assumed to be defined at the cell center or at the cell interface while $Y$ is supposed to be defined at the cell edge. $Z$ is actually the radius $r$. Note that we distinguish two velocities $U_{i}^{n+1}$ and $u_{I}^{n+1}$ and two pressures $p_{i}^{n+1}$ and $p_{I}^{n+1}$, the latter of which are determined from the linearized Riemann problems which are evaluated at the time step " $n+1$ ", where the values of the dependent variables should be solved. That is, we consider the Riemann problems at each cell interface " $I^{\prime \prime}$ at each time step " $n+1$ ", the left and right constant states of which are given by $\left(\tau_{i}^{n+1}, U_{i}^{n+1}, p_{i}^{n+1}\right)$ and $\left(\tau_{i+1}^{n+1}, U_{i+1}^{n+1}, p_{i+1}^{n+1}\right)$, respectively. We solve these problems approximately by linearizing the advection equations as described below. It should be noted again that these constant states are evaluated at the time step " $n+1^{\prime \prime}$, so that we cannot know them a priori. Hence we must solve these Riemann problems at each iteration step of Newton-Raphson methods. The physical meaning of the interface values obtained in this way is rather obscure compared with the counterparts in explicit schemes, since the exact solution of the Riemann problem describes the interaction of the nonlinear wave after the time of the initial condition (the time step " $\left.n+1^{\prime \prime}\right)$. The interpretation here is that they approximate the states after the interactions of nonlinear waves during the time steps " $n$ " to " $n+1$ ". Practically, these values are given as follows. If we pay attention only to the advection terms, then we get

$$
\frac{\partial}{\partial t}\left\{\begin{array}{c}
\tau \\
U \\
p
\end{array}\right\}=\left(\begin{array}{ccc}
0 & \frac{e^{\phi}}{\Gamma} 4 \pi r^{2} & 0 \\
0 & 0 & -\frac{e^{\phi} \Gamma}{h} 4 \pi r^{2} \\
0 & -\gamma \frac{p}{\tau} \frac{e^{\phi}}{\Gamma} 4 \pi r^{2} & 0
\end{array}\right) \frac{\partial}{\partial m}\left\{\begin{array}{c}
\tau \\
U \\
p
\end{array}\right\}
$$

where $\gamma$ is the adiabatic index and is defined as:

$$
\gamma \equiv\left(\frac{\partial \ln p}{\partial \ln \rho_{b}}\right)_{s} \quad(s \text { is the entropy per baryon })
$$

In equation (35), the dependent variable is changed from $\varepsilon$ to $p$, for two reasons. Firstly, the eigen vectors are very simple in this representation: 


$$
\begin{aligned}
& \lambda_{1}=-e^{\phi} 4 \pi r^{2} \rho_{b} c_{s} \quad \boldsymbol{r}_{\mathbf{1}}=\left(\begin{array}{c}
\tau \\
\Gamma c_{s} \\
-\gamma p
\end{array}\right) \quad \boldsymbol{l}_{\mathbf{1}}=\left(\begin{array}{c}
0 \\
\frac{1}{2 \Gamma c_{s}} \\
\frac{1}{2 \gamma p}
\end{array}\right) \\
& \lambda_{2}=0 \quad \boldsymbol{r}_{2}=\left(\begin{array}{c}
1 \\
0 \\
0
\end{array}\right) \quad \boldsymbol{l}_{2}=\left(\begin{array}{c}
1 \\
0 \\
\tau \\
\gamma p
\end{array}\right) \\
& \lambda_{3}=e^{\phi} 4 \pi r^{2} \rho_{b} c_{s} \quad \boldsymbol{r}_{\mathbf{3}}=\left(\begin{array}{c}
\tau \\
-\Gamma c_{s} \\
-\gamma p
\end{array}\right) \quad \boldsymbol{l}_{\mathbf{3}}=\left(\begin{array}{c}
0 \\
-\frac{1}{2 \Gamma c_{s}} \\
-\frac{1}{2 \gamma p}
\end{array}\right),
\end{aligned}
$$

where $\lambda$ 's are the eigen values, and $\boldsymbol{r}$ 's and $\boldsymbol{l}$ 's are the corresponding right and left eigen vectors. In this equations, $c_{s}$ is the sound velocity defined as:

$$
c_{s} \equiv \sqrt{\gamma \frac{p}{\rho_{b} h}} .
$$

As in the Newtonian hydrodynamics, there are three modes, two of which correspond to the left- and right-going sound waves and the rest of which represents the contact discontinuity. In this representation, it is clear that pressure and velocity are continuous across the contact discontinuity. This feature is taken over to the solution of the linearized Riemann problems when these eigenvectors are used to solve them. It is not the case when other dependent variables are chosen. This is the second reason to use these variables. In order to linearize equation (35) we just replace the Jacobian matrix by some constant matrix at each cell interface. In this paper, arithmetically averaged $\rho_{b m} \equiv\left(\rho_{b L}+\rho_{b R}\right) / 2, U_{m} \equiv\left(U_{L}+U_{R}\right) / 2$ and $\varepsilon_{m} \equiv\left(\varepsilon_{L}+\varepsilon_{R}\right) / 2$ are used to evaluate the constant matrix, where the subscripts $L$ and $R$ mean that they are evaluated at the left and right constant states of each Riemann problem, respectively. The eigen values and the corresponding left and right eigen vectors given above are also evaluated in terms of these averaged variables at each cell interface. The linearized advection equations thus obtained can be solved analytically. $u_{I}$ and $p_{I}$ evaluated in this way prove to be as follows:

$$
\begin{aligned}
& u_{I}=\frac{1}{2}\left(u_{R}+u_{L}\right)-\frac{c_{s m}}{2 \gamma_{m} p_{m}}\left(p_{R}-p_{L}\right) \\
& p_{I}=\frac{1}{2}\left(p_{R}+p_{L}\right)-\frac{\gamma_{m} p_{m}}{2 c_{s m}}\left(u_{R}-u_{L}\right),
\end{aligned}
$$

where the subscript $m$ means they should be evaluated by using the above averaged variables. It should be noted that $p_{m}$ is different from the arithmetic mean of $p_{L}$ and $p_{R}$. 
As is clear, both $u_{I}$ and $p_{I}$ are the arithmetic mean of the left- and right-state values with the correction terms which are proportional to $\left(p_{R}-p_{L}\right)$ and $\left(u_{R}-u_{L}\right)$, respectively. These correction terms serve as a numerical diffusion so that no artificial viscosity is necessary. The above representations of the velocity and the pressure at the cell interface are so simple that it is quite easy to evaluate their derivatives with respect to the dependent variables in the course of the Newton-Raphson scheme.

In the present code, in order to achieve spatial second order accuracy, the piecewise linear distributions of dependent variables $\rho_{b}, U, \varepsilon$ and $\Gamma$ are introduced. It is well known that some limiting procedure is necessary to maintain the monotonicity of the numerical solution. In this paper, we use the simplest slope prescription: if $\boldsymbol{s}_{l}$ and $\boldsymbol{s}_{r}$ denote the left- and right-hand difference quotients of the above dependent variables, the slopes $\boldsymbol{S}$ are determined by the formula

$$
S^{i}= \begin{cases}s_{l}^{i} & \text { for }\left|s_{l}^{i}\right| \leq\left|s_{r}^{i}\right| \text { and } s_{l}^{i} \cdot s_{r}^{i}>0 \\ s_{r}^{i} & \text { for }\left|s_{l}^{i}\right|>\left|s_{r}^{i}\right| \text { and } s_{l}^{i} \cdot s_{r}^{i}>0 \\ 0 & \text { otherwise }\end{cases}
$$

where the superscript $i$ distinguishes the dependent variables $\rho_{b}, U, \varepsilon$ and $\Gamma$. This prescription will need some improvement in the future.

\subsection{Numerical Implementation of the Implicit Scheme}

The above finite differenced equations (22) through (31) with the definitions of interface values (41) and (42) form the system of the nonlinearly coupled simultaneous equations with respect to the dependent variables $\tau, U, \varepsilon, Y_{e}, r, \lambda, \Gamma, \phi, \tilde{m}$ and $h$. In order to solve these equations, the standard Newton-Raphson iteration scheme is utilized for the linearized equations. That is, if we define the dependent variables vector as

$$
\boldsymbol{X}=\left(\cdots, \tau_{i}, U_{i}, \varepsilon_{i}, Y_{e i}, r_{I}, \lambda_{i}, \Gamma_{i}, \phi_{I}, \tilde{m}_{I}, h_{i}, \cdots\right)^{t}
$$

where the subscripts $i$ and $I$ mean the $i$-th mesh and interface values, and denote all the equations in the vector representations as

$$
\boldsymbol{F}(\boldsymbol{X})=0
$$

then the linearized equations become

$$
\boldsymbol{F}(\tilde{\boldsymbol{X}})+\frac{\partial \boldsymbol{F}(\tilde{\boldsymbol{X}})}{\partial \tilde{\boldsymbol{X}}} \cdot \delta \boldsymbol{X}=0
$$


where $\tilde{\boldsymbol{X}}$ is some guess for the correct $\boldsymbol{X}$. This linearized equation (46) is solved repeatedly while improving the guess by

$$
\tilde{\boldsymbol{X}}_{\text {new }}=\tilde{\boldsymbol{X}}+\delta \boldsymbol{X}
$$

until the error $|\delta \boldsymbol{X}|$ becomes less than some small value which is set to $10^{-5}$ in the following calculations. As is clear, the Jacobian matrix $\frac{\partial \boldsymbol{F}(\tilde{\boldsymbol{X}})}{\partial \tilde{\boldsymbol{X}}}$ turns out to be a block-tridiagonal matrix whose block size is $10 \times 10$. At each step of the above iteration, the linearized equations (46) are solved by the standard Gauss elimination scheme, though other elaborate schemes can be applied to this type of matrix. Note, however, that the matrix for the hydrodynamic sector is very small compared with that for neutrinos. The implementation of such an efficient solver will be done at the next step, where the neutrino transfer code will be combined.

As stated above, the formulae for the velocity and the pressure at the cell interface (equation (41) are so simple that we can calculate their derivatives with regard to the dependent variables analytically. In so doing, however, the contributions from the sloping prescription are dropped, which does not cause serious problems for convergence of the Newton-Raphson iteration process. The thermodynamic derivatives are obtained by differencing the EOS table numerically, except for the adiabatic index $\gamma$, which is stored in the table.

\subsection{Time-Step Control}

The time step is determined so that the change of any dependent variables should not exceed an appropriate upper limit, which is chosen to be $2 \%$ in most of the following calculations, at each time step. In order to save the computation time, however, we correct the next time step and don't repeat the same time step even though the above criterion is not satisfied at some time step. In fact, if the change of some variable exceeds the above criterion, the subsequent time step is decreased by a factor of 0.95 . On the other hand, if none of the variations of the dependent variables exceeds the upper limit, then we multiply the next time step by 1.05. The time variation of the Courant number which is defined as:

$$
\text { Courant number } \equiv \frac{\Delta t}{\left(\frac{\Delta x}{c_{s}+|U|}\right)}
$$

is monitored for all the calculations and is shown for some test calculations below. 


\section{TEST CALCULATIONS}

In the following, some results for the representative test problems are shown in order to clarify the performance of the present hydrodynamical code. Among them there are shock tube problems both for non-relativistic and special relativistic cases, Sedov's point explosion problem in the uniform matter, Oppenheimer-Snyder's dust collapse problem and the non-relativistic self-similar collapse of a $\gamma=4 / 3$ polytrope. Moreover, the results of the hydrostatic calculations and the simple adiabatic collapse simulations are demonstrated in order to judge whether the present code can be applied to the simulation for the late time stage of the collapse-driven supernovae. In order to compare the results, the parameter sets adopted here for the first five problems are almost the same as those studied by Swesty. In the course of these simulations no special tuning to the specific problem is done except for the boundary conditions, the mesh system and the equation of state.

\subsection{The Non-Relativistic Shock Tube Problem}

The most standard parameter set for this problem is that proposed by Sod (1978):

$$
\left\{\begin{array} { l } 
{ \rho _ { L } = 1 } \\
{ p _ { L } = 1 } \\
{ U _ { L } = 0 }
\end{array} \quad \left\{\begin{array}{l}
\rho_{R}=0.125 \\
p_{R}=0.1 \\
U_{R}=0
\end{array}\right.\right.
$$

where the subscripts $L$ and $R$ mean the left- and right-state values and CGS units are assumed. The equation of state for this problem is $p=(\gamma-1) \rho_{b} \varepsilon$ with $\gamma=4 / 3$ and $5 / 3$.

Since the present code assumes spherically symmetric geometry, we formulate the problem, which is originally for plane symmetric geometry, over a thin shell whose curvature is safely ignored. Following Swesty, we take $R=10^{4} \mathrm{~cm}$ and $\delta R=2 \mathrm{~cm}$ for the radius and the thickness of the thin shell, respectively, and we define $x=R-r$ and specify the region $-1<x \leq 0$ as the left state. 100 uniform meshes are used. Although the calculations have been done both for $\gamma=4 / 3$ and $5 / 3$, only the results for $\gamma=5 / 3$ are described since the features of the numerical solutions are nearly the same both qualitatively and quantitatively.

In figure 1 the representative distributions of $\rho_{b}, U, p$ and $\varepsilon$ for $\gamma=5 / 3$ are shown with the corresponding analytic solutions. The time is $0.675 \mathrm{sec}$ (400 time steps). As can be seen, the shock is resolved by 3 meshes in the present case. Moreover, no oscillations occur behind the shock and the rarefaction wave. The Rankine-Hugoniot condition is strictly satisfied without any artificial viscosity. However, we can see the small jump of the specific 
internal energy density ahead of the contact surface. This is not an oscillation but an erroneous contact surface. This can be understood from the smoothness of the velocity and the pressure at the contact surface. The approximate solution of the Riemann problem is least accurate at the first time step, when the largest discontinuities exist. The observed incorrect jump of the specific internal energy density at the contact surface is the result of the inaccurate approximate solution at the first time step. Since this scheme is Lagrangian, the contact discontinuity is never smeared out at all once it is formed. However, this is not a serious problem in the simulation of the realistic core collapse, since even the contact surface between the iron core and the mantle spreads over a considerable distance and is not a strict discontinuity.

The time steps for these calculations are controlled so that the maximum variation of any dependent variables does not exceed $2 \%$. As a result, the Courant number defined earlier is kept as large as 0.3 through the calculations. The calculation which allows the maximum variation of $15 \%$ has also been done in order to see the effect of time step control. The result shows the tendency to smear out the shock and the rarefaction wave. However, the effect is not so large in the non-relativistic case. This issue will be raised again in the next section.

\subsection{The Special Relativistic Shock Tube Problem}

Next shown are the numerical solutions for the following Riemann problem:

$$
\left\{\begin{array} { l } 
{ \rho _ { L } = 0 . 2 } \\
{ p _ { L } = 2 \times 1 0 ^ { 1 8 } } \\
{ U _ { L } = 0 }
\end{array} \quad \left\{\begin{array}{l}
\rho_{R}=0.1 \\
p_{R}=1 \times 10^{18} \\
U_{R}=0
\end{array},\right.\right.
$$

where the units are again CGS. This parameter set is the same as that used by Swesty. We use 100 zones with an equal spatial resolution also in this case. The equation of state is again $p=(\gamma-1) \rho_{b} \varepsilon$.

Figure 2 shows the distributions of density, velocity, pressure and specific internal energy density at the time of $3.03 \times 10^{-10} \mathrm{sec}$ (490 numerical steps). $\gamma=5 / 3$ also in this case. As can be seen clearly, the Rankine-Hugoniot condition at the shock wave is satisfied very well. No oscillation occurs in this case, either, except for the one mesh just ahead of the contact discontinuity. The cause of this erroneous jump is the same as for the non-relativistic shock tube. Since the initial discontinuity of the density is smaller in this case than in the previous case, the resulting error becomes smaller. One thing to be mentioned here is that the upper limit of the variation of the dependent variables is 
chosen to be $0.5 \%$ in this calculation so that the shock can be resolved in a few zones. The resultant Courant number is $\sim 0.2$. In order to see the effect of time steps on the resolution of the numerical solution, we have done two other simulations varying the upper limit. In figure 3 we show the results for the upper limit of $2 \%$. The Courant number is $\sim 2$ through the integration. As can be clearly seen, both the shock wave and the rarefaction wave are smeared out considerably. This trend is much more remarkable in figure 4, which shows the results for an upper limit of $10 \%$ and the Courant number is as large as 8 at the end of the calculation. It should be also noted that in this calculation the Courant number is set to be 3 from the beginning. From these results it is concluded that although the present implicit scheme is stable for the Courant number $>1$, in order to keep the resolution of the solution the maximum variation of the dependent variables must be chosen so that the Courant number should be $\sim 0.2$ at the shock wave. However, note that this does not mean that this code cannot not be applied to the realistic simulation of the collapse-driven supernovae, since what we should overcome is not the Courant limit at the shock wave but the limit at the nascent neutron star. This issue will be discussed again in the section of adiabatic collapse calculation.

\subsection{Sedov's Point Explosion in a Uniform Medium}

The dimensional parameters which characterize the problem are the density of the uniform matter $\rho_{0}$ and the total energy of explosion $E_{\text {tot }}$. The problem, however, can be formulated in the completely non-dimensional form. Hence the solutions with different initial parameters are equivalent to each other. We choose the following parameters for simplicity:

$$
\left\{\begin{array}{c}
\rho_{0}=1 \\
E_{\text {tot }}=1 \\
\gamma=5 / 3
\end{array}\right.
$$

where the units are CGS. The computational region is the sphere with the radius of 1 , and is covered by 100 equal baryon mass zones. The equation of state is $p=(\gamma-1) \rho_{b} \varepsilon$. The calculation is initiated by depositing the total energy in the central mesh. For numerical convenience the uniform ambient matter has the specific internal energy density of $\sim 10^{-3} \mathrm{~cm}^{2} / \mathrm{sec}^{2}$. This is justified since the ambient specific internal energy density is negligibly small compared with that of shocked matter. The results are shown in figure 5 . The shock is resolved by $2 \sim 3$ meshes also in this case, and the analytic solution is reproduced quite well in most of the computational region. In the central $\sim 10$ meshes, however, the numerical solution is not very good. This is because the mesh for this problem is uniform in baryon mass and the spatial resolution is not sufficient for the central region. 


\subsection{The Self-Similar Collapse of a $\gamma=4 / 3$ Polytrope}

It is well known that a non-relativistic gas with an adiabatic index of $\gamma=4 / 3$ is neutrally stable against gravitational collapse. Goldreich \& Weber (1980) found a solution of a self-similarly collapsing gas with $\gamma=4 / 3$. Yahil (1983) generalized this solution to the collapse for different $\gamma$ 's. These self-similar solutions approximate the homologous collapse of the inner core of the collapse-driven supernovae. Hence it is indispensable to the numerical schemes for the collapse-driven supernovae that they can reproduce this self-similar collapse.

The initial condition is made as follows: assuming the polytropic equation of state $p=K \rho_{b}^{\gamma}$ with $\gamma=4 / 3$, we first solve the Lane-Emden equation. Here we take $K$ as the value for the relativistically degenerate gas with electron fraction $Y_{e}=0.45$. The central density is set to be $1.0 \times 10^{8} \mathrm{~g} / \mathrm{cm}^{3}$. Then we take out the inner $1 \mathrm{M}_{\odot}$ and reduce the internal energy uniformly so that the total energy becomes 0 . This reduction factor is nearly equal to the pressure deficit $d$ defined by Goldreich-Weber as

$$
d \equiv\left(\frac{M_{\mathrm{hc}}\left(=1.0 \mathrm{M}_{\odot}\right)}{M_{0} \times 1.0449}\right)^{\frac{2}{3}},
$$

where $M_{\mathrm{hc}}$ and $M_{0}$ are the homologous core mass which is set to be $1 \mathrm{M}_{\odot}$ in this model and the mass of the polytrope without pressure deficit, respectively.

The computational region $\left(1 \mathrm{M}_{\odot}\right)$ is covered with 100 equal baryon mass zones also in this case. It is noted that the equation of state for simulation is ideal gas like $\left(p=(\gamma-1) \rho_{b} \varepsilon\right)$ with $\gamma=4 / 3$, not adiabatic $\left(p=K \rho_{b}^{\gamma}\right)$. This is because we want to see how well the constancy of entropy is maintained in the course of the numerical integration.

The results are shown in figure 6 , where the time variation is presented for density, velocity, pressure and the quantity proportional to entropy: $p / \rho_{b}^{\gamma}$. In this figure, the solid lines are just connecting the data for the same time step. When we pay attention to the velocity distribution, it is clear that most parts of the polytrope collapse homologously, that is, the infalling velocity is proportional to the radius. Although it is not the case for the outer few zones, this is due to the boundary condition which is set by hand and is not consistent with the actual dynamics. In the actual simulation of the supernovae, this problem concerning the outer boundary condition should be avoided by locating the boundary at a sufficiently distant position. On the other hand, it is also found that the entropy distribution remains almost constant for most parts of the polytrope through the evolution. The exception is the outer few zones also in this case. Ignoring these outer parts, we can find the maximum error of the entropy is about $8 \%$ at the innermost zone and about $5 \%$ for the other zones. The calculation is terminated when the central density reaches 
$4.85 \times 10^{12} \mathrm{~g} / \mathrm{cm}^{3}$ after 600 time steps. The relativistic correction is $g_{00} \sim 0.97$ at that time.

\subsection{Oppenheimer-Snyder's Dust Collapse Problem}

The general relativistic collapse of uniform pressureless matter was analytically solved by Oppenheimer \& Snyder. When we assume the matter is initially $(\bar{t}=0)$ at rest with the uniform density $\rho_{b 0}$ and the radius $r_{0}$, then the subsequent motion is described parametrically as follows:

$$
\begin{aligned}
r & =\frac{r_{0}}{2}(1+\cos \eta) \\
\bar{t} & =\frac{1}{2} \sqrt{\frac{3}{8 \pi G \rho_{b 0}}}(\eta+\sin \eta),
\end{aligned}
$$

where $\eta$ is varied from 0 to $\pi$. It should be mentioned that the above $\bar{t}$ is the proper time for each mass element and should be distinguished from the coordinate time $t$. The boundary condition for the coordinate time $t$ is not altered in this simulation, they are related to each other by

$$
\frac{d t}{d \bar{t}}=e^{-\phi} .
$$

The time coordinate in figure 7 is the coordinate time $t$ which is obtained by numerically integrating equation (53). However, the difference is not significant except for the final few msec. Note also that in this time coordinate it takes infinite time for the mass shell to reach the event horizon. However, it is not a serious problem, as stated earlier, since the main purpose of this code is to study the process to produce a neutron star.

Following Swesty, we take $2 \mathrm{M}_{\odot}$ cloud with a uniform density of $\rho_{b 0}=10^{8} \mathrm{~g} / \mathrm{cm}^{3}$ for the initial model. Since the present code cannot handle zero-pressure, we use the polytropic equation of state $p=K \rho_{b}^{\gamma}$ with $\gamma=4 / 3$ and negligibly small $K$. This initial configuration is covered with 160 equal baryon mass meshes. The calculation is terminated after 3000 time steps when the central density reaches $1.72 \times 10^{14} \mathrm{~g} / \mathrm{cm}^{3}$.

In figure 7 , the motions of the representative mass elements are shown. The solid lines are exact solutions in this case. As shown in this figure, the coincidence between the numerical solution and the exact solution is satisfactory. The slight difference (less than $1 \%$ in time) appears only for the last few msec which is not clear in figure 7 . This is partially due to the error of the integration of the coordinate time (equation (53)). The above result along with the next one clearly shows that the present code can treat the general relativistic gravity correctly. 


\subsection{The Hydrostatic Calculation}

This and the next section are devoted to demonstrate the performance of this code in the context of the collapse-driven supernovae, that is, it can calculate the hydrodynamics far beyond the dynamical time scale after the proto-neutron star is formed in the central region. This is the crucial point for the purpose of this paper.

In this section, we take a hydrostatic configuration for the initial condition for the dynamical calculation, and see whether this initial state will be maintained or not. For that purpose, we first solve the Tolman-Oppenheimer-Volkov equations:

$$
\begin{aligned}
\frac{d p}{d r} & =-(\rho+p) \frac{\tilde{m}(r)+4 \pi r^{3} p}{r^{2}\left(1-\frac{2 \tilde{m}(r)}{r}\right)} \\
\frac{d \phi}{d r} & =\frac{\tilde{m}(r)+4 \pi r^{3} p}{r^{2}\left(1-\frac{2 \tilde{m}(r)}{r}\right)} \\
\rho & =\rho_{b}(1+\varepsilon) \\
\tilde{m}(r) & =\int_{0}^{r} \rho\left(r^{\prime}\right) 4 \pi r^{\prime 2} d r^{\prime},
\end{aligned}
$$

where the notation is the same as in the dynamical equations. The boundary condition for $\phi$ is consistent with the dynamical counterpart:

$$
e^{\phi_{s}}=\left(1-\frac{2 \tilde{m}_{s}}{r_{s}}\right)^{\frac{1}{2}}
$$

The subscript $s$ in this equation means the core surface values. The equation of state is assumed to be polytropic $p=K \rho_{b}^{\gamma}$ with $K=1.97 \times 10^{-3}$ in the CGS units and $\gamma=2.5$. The central density is taken to be $\rho_{b c}=4 \times 10^{14} \mathrm{~g} / \mathrm{cm}^{3}$. The initial model thus obtained has the gravitational mass of $\tilde{M}_{s}=1.72 \mathrm{M}_{\odot}$ and the radius of $R_{s}=7.7 \mathrm{~km}$. This approximate neutron star is nearly the same as that used by Schinder et al (1988).

In the dynamical simulation, we assume the same equation of state and use 100 uniform baryon mass zones. In figures 8 and 9 the trajectories of all the mass zones are shown with the time variation of the Courant number. The time step is controlled so that all the dependent variables should not change more than $2 \%$ at each time step. Figure 8 shows the mass element motions for the first $10 \mathrm{msec}$. As can be seen, nothing occurs except for the very little oscillation during the first $1 \mathrm{msec}$. The Courant number is shown with a curved line across the trajectories. It is found that the time step is increased monotonically. 
This is because the matter velocities decrease monotonically after the first small transient. Figure 9 shows the mass trajectories for the same model but for a much longer time scale. The final time is 20sec, which should be compared with the dynamical time scale of the neutron star $\lesssim 1 \mathrm{msec}$. It is also noted this time corresponds to only 300 numerical time steps. The final Courant number exceeds $10^{6}$. These results imply that this dynamical calculation reproduced the hydrostatic configuration correctly and also guarantee that this code treats the general relativity appropriately.

\subsection{The Adiabatic Collapse Calculation}

Finally shown are the results of the model calculations of the adiabatic core collapse. In these simulations, we assume the simplified equation of state which approximately reproduces the realistic simulations with detailed micro physics and neutrino transfer, and calculate the collapse of the iron core. The phenomenological equation of state adopted here is what Takahara \& Sato (1982) used for their parametric research of the dynamics of core collapse. The total pressure $p_{t o t}$ consists of the cold part $p_{c}$ and the thermal part $p_{t}$ as follows:

$$
\begin{aligned}
p_{t o t}\left(\rho_{b}, \varepsilon_{t}\right) & =p_{c}\left(\rho_{b}\right)+p_{t}\left(\rho_{b}, \varepsilon_{t}\right) \\
p_{c}\left(\rho_{b}\right) & =K \rho_{b}^{\Gamma} \\
p_{t}\left(\rho_{b}, \varepsilon_{t}\right) & =\left(\gamma_{t}-1\right) \rho_{b} \varepsilon_{t},
\end{aligned}
$$

where $\varepsilon_{t}$ is the specific thermal energy density, and the adiabatic index $\Gamma$ is assumed to be density dependent. Actually $\Gamma=4 / 3$ for $\rho_{b} \leq 4 \times 10^{9} \mathrm{~g} / \mathrm{cm}^{3}$ and $10^{12} \leq \rho_{b} \leq 2.8 \times 10^{14} \mathrm{~g} / \mathrm{cm}^{3}$, and $\Gamma=2.5$ for $\rho_{b} \geq 2.8 \times 10^{14} \mathrm{~g} / \mathrm{cm}^{3}$. The value of $\Gamma$ for $4 \times 10^{9} \leq \rho_{b} \leq 10^{12} \mathrm{~g} / \mathrm{cm}^{3}$ is the model parameter. Similarly, the thermal stiffness $\gamma_{t}$ is varied from model to model. By adjusting these parameters, we can get both successful explosion models and stalled shock models. In fact, larger values help the shock propagation and tend to strengthen the explosion. The initial models are Woosley's $15 \mathrm{M}_{\odot}$ and $35 \mathrm{M}_{\odot}$ precollapse models (Woosley 1990). In the former model, the central $1.32 \mathrm{M}_{\odot}$ iron core is covered by 100 non-uniform mass zones in which the baryon mass of each cell is increased outward. $\Gamma=1.30$ and $\gamma_{t}=1.30$ are assumed. This model explodes in a prompt way. In the latter model, on the other hand, the central $2.0 \mathrm{M}_{\odot}$ is covered with 150 non-uniform meshes and $\Gamma=1.28$ and $\gamma_{t}=1.25$ are assumed. The shock is stalled inside the iron core for this model as shown below.

Figures 10 and 11 show all the mass trajectories for the $15 \mathrm{M}_{\odot}$ model with the time variation of the Courant number. Again I will show the results in two different time scales. 
In figure 10, we can see the motions of mass elements until the shock reaches the iron core surface. As stated above, since the shock propagates the entire iron core without stagnation and some part of the outer core moves outward at the break out, this model is a successful prompt explosion model. It is also noted that the central part becomes almost hydrostatic. The data concerning the location of each mass element and the Courant number are saved every 10 time steps. As can be seen, the Courant number increases up to about 500 at the break out. Figure 11 shows the subsequent evolution until the calculation is terminated.

The final time is $600 \mathrm{msec}$ and corresponds to 5000 numerical time steps. Since the mantle is not taken into consideration, the result after shock breaks out is not of physical significance. However, the calculation was continued in order to see how large the Courant number gets. As can be seen, the final Courant number exceeds 1000. One thing to be mentioned here is that the Courant number shown here is determined not at the shock wave but at the center of the proto-neutron star, which implies that we could overcome the severe Courant limit at the center without instability. This seems quite encouraging for the purpose of this project. It should be mentioned, however, that this is partly because there is no significant accretion on the proto-neutron star in the successful explosion model. Hence it is of greater importance to see the time evolution of the stalled shock model.

In figure 12 we can see the motions of the representative mass elements for the $35 \mathrm{M}_{\odot} \odot$ model until 50msec after the core bounce. In this case, the shock does not expel the accreting matter, that is, the shock is stalled. The inner core becomes nearly hydrostatic also in this case. However, since the matter is still falling onto the proto-neutron star continuously, the Courant number cannot become so large as the previous model as expected. The subsequent evolution is shown in figure 13. The final time is $900 \mathrm{msec}$ after the start of the simulation. The total number of numerical time steps is 8000 . As can be seen, in this case the Courant number also becomes as large as 1000. Again this is a very encouraging result for this project. However, it should be mentioned that the resolution of the outer core is not sufficient to resolve the shock wave for the $35 \mathrm{M}_{\odot}$ model. This is not only because the variation of dependent variables is allowed up to $2 \%$ in this calculation (see the results of the relativistic shock tube problems), but also because matter continues to concentrate to the center. Hence we must implement some rezoning routine or we should use a larger number of zones. This is the problem to be solved in the next step.

\section{SUMMARY}

The implicit general relativistic hydrodynamical scheme is coded using the approximate Riemann solver and extensive numerical tests are accomplished. The scheme is very simple 
since the formulae to evaluate the velocity and the pressure at the cell interface have such simple forms that we can easily calculate their derivatives with regard to the dependent variables. The numerical solutions obtained by this scheme show no oscillation after both shock waves and rarefaction waves unlike the schemes requiring an artificial viscosity, although a small erroneous jump appears after the contact surface. The calculations of the simple adiabatic collapse of massive stellar cores indicate this code is quite stable even if we take a much longer time step than the Courant time step, which fact is very encouraging for its application to the more realistic calculation of the late time explosion. However, it is also found that the time step should be adjusted so that the Courant number at the shock wave should be $\sim 0.2$. It is also probable some automatic rezoning routine should be implemented to resolve the shock wave sufficiently all through the calculation.

The purpose of this project is to provide a tool to study the radiation hydrodynamics of supernova cores far beyond the dynamical time scale of the neutron star. We are now proceeding to the next step, in which the present hydrodynamic code is combined with a neutrino transfer code using the multi-energy group flux-limited diffusion scheme. However, the final goal of this project is to make a Boltzmann solver and combine it with this implicit hydrodynamical code thereby removing uncertainties regarding the neutrino transfer.

In recent years, the importance of multi-dimensional effects on the supernova explosion has been pointed out and many detailed numerical simulations have been done. At present, it seems that the clues to the successful supernova explosion are such multi-dimensional effect and neutrinos. Although some effort has been made to do the simulation of multidimensional radiation transfer, it seems still far from satisfaction. Hence the one-dimensional simulations with detailed neutrino transfer and multi-dimensional simulations will play complementary roles to one another. It is also possible that one-dimensional simulation would serve as a guide to make some approximate treatment of the multi-dimensional neutrino transfer.

I am grateful to S. Woosley for providing us with the details of his progenitor models. I also thank H. Suzuki for helpful advice and comments and W. Hillebrandt, E. Müller and E. Skillman for their kind review of the manuscript. The numerical calculations were mainly done on the workstations of the theoretical astrophysics group of the University of Tokyo, the national institute for high energy physics (KEK) and Max-Planck-Insitut für Astrophysik. This work was partially supported by the Grant-in-Aid for Scientific Research from the ministry of Education, Science and Culture of Japan Nos. 05243103, 04234104, 07740344 and by Japan Society for the Promotion of Science (JSPS) Postdoctoral Fellowships for Research Abroad. 


\section{REFERENCES}

Bethe, H. A. 1990, Rev. Mod. Phys., 62, 801

Bowers, R. L., \& Wilson, J. L. 1991, Numerical Modeling in Applied Physics and Astrophysics (Boston:Jones \& Bartlett)

Burrows, A., Hayes, J., \& Fryxell, B. A. 1995, ApJ, 450, 830

Colgate, S. A., \& White, R. H. 1966, ApJ, 143, 626

Eulderink, F., \& Mellema, G. 1995, A\&AS, 110, 587

Goldreich, P., \& Weber, S. V. 1980, ApJ, 238, 991

Herant, M., Benz, W., Hix, R., Fryer, C. L., \& Colgate, S. A. 1994, ApJ, 435, 339

Hirsch, C. 1990, Numerical Computation of Internal and External Flows (Chichester:John Wiley \& Sons)

Janka, H. -T., \& Müller, E. 1995, A\&A in press

May, M. M., \& White, R. H. 1967, Methods Compt. Phys., 7, 219

Müller, E. 1991, Lecture Notes in Physics, 373, 97

Romero, J. V., Ibáñez, J. M., Martí, J. M., \& Miralles, J. A. 1995, submitted to ApJ

Schinder, P. J., Bludman, S. A., \& Piran, T. 1988, Phys. Rev. D, 37, 2722

Shimizu, T., Yamada, S., \& Sato, K. 1994, ApJ, 432, L119

Sod, G. A. 1978, J. Comp. Phys., 27, 1

Suzuki, H. 1990, Ph.D. thesis, Univ. of Tokyo

Swesty, F. D. 1995, ApJ, 445, 811

Takahara, M., \& Sato, K. 1982, Prog. Theor. Phys., 68, 795

Woosley, S. E. 1990, private communication

Yahil, A. 1983, ApJ, 265, 1047 
Fig. 1. - The distribution of density, velocity, pressure and specific internal energy density at $t=0.675 \mathrm{sec}$ (400 time steps) are shown with the analytic solutions (solid lines) for $\gamma=5 / 3$.

Fig. 2.- The distribution of density, velocity, pressure and specific internal energy density for the special relativistic shock tube problem described in the text at $t=3.03 \times 10^{-10} \mathrm{sec}$ (490 time steps) are shown with the analytic solutions (solid lines) for $\gamma=5 / 3$.

Fig. 3.- The numerical solution for the same Riemann problem as Fig. 2 but with larger time step (the Courant number $=2.0) . t=2.95 \times 10^{-10} \mathrm{sec}(60 \mathrm{steps})$.

Fig. 4. - The numerical solution for the same Riemann problem as Fig. 2 but with much larger time steps (the Courant number reaches $\sim 8.0$ at the end of the calculation). $t=2.80 \times 10^{-10} \sec (16$ steps $)$.

Fig. 5.- The numerical solution for Sedov's point explosion problem described in the text. The time is $0.49 \mathrm{sec}$ and the step number is 10000 . The solid lines are the exact solutions. $\gamma=5 / 3$.

Fig. 6. - The numerical solution for the self-similar collapse of a $\gamma=4 / 3$ polytrope. The distributions are shown every 100 steps. The times are $0.463,0.700,0.804,0.881,0.920$, 0.937 seconds. The solid lines are just connecting the data for the same time step.

Fig. 7.- The trajectories of the representative mass elements for the Oppenheimer-Snyder dust collapse problem are shown with white circles. The solid lines are exact solutions. The baryon masses for the trajectories are $0.125,0.375,0.625,0.875,1.125,1.375,1.625$, $1.875 \mathrm{M}_{\odot}$.

Fig. 8. - The mass trajectories for the hydrostatic problem described in the text. The curved lines across the trajectories show the time variation of the Courant number. The evolution for only the first $10 \mathrm{msec}$ is shown.

Fig. 9.- The same as Fig. 8 but for the whole calculation.

Fig. 10. - The mass trajectories for the adiabatic collapse of the $15 \mathrm{M}_{\odot}$ model. The line across the trajectories show the time variation of the Courant number. The evolution only for the first $170 \mathrm{msec}$ is shown.

Fig. 11. - The same as Fig. 10 but for the whole calculation.

Fig. 12. - The mass trajectories for the adiabatic collapse of the $35 \mathrm{M}_{\odot}$ model. The line across the trajectories show the time variation of the Courant number. The evolution only for the first $400 \mathrm{msec}$ is shown. 
Fig. 13. - The same as Fig. 12 but for the whole calculation. 